\title{
What does research on nicotine and tobacco use have to offer alcohol reseachers?
}

\author{
OVIDE F. POMERLEAU, Ph.D.
}

\begin{abstract}
Professor of Psychology in Psychiatry and Director, Behavioral Medicine Program, University of Michigan School of Medicine, Riverview Building, 900 Wall Street, Ann Arbor, MI 48105, $U S A$
\end{abstract}

What does research on nicotine and tobacco use have to offer that is relevant and possibly useful to an alcohol researcher? First and foremost, nicotine and tobacco research may provide some helpful insights into addiction processes and substance use treatment in general. Smoking can be seen as a model disorder, not only because of its prevalence and its importance as a public health problem, but because the consistency of smoking behavior and the frequency of dosing (nicotine has a short addictive cycle) allow human experimentation in the laboratory without compromising relevance. ${ }^{1}$ Recent technological developments in the topographical analysis of smoking behavior and in the assay of nicotine in biological substrates have facilitated both laboratory and field research, and comprehensive biobehavioral explanations of smoking/nicotine dependence have been proposed. ${ }^{2}$ Increasingly sophisticated public health campaigns have produced a dramatic decrease in smoking, with the level of smoking in the United States down from over $60 \%$ of the adult population in the early 1960 's to fewer than $30 \%$ in the late 1980 's. Over the same time period, therapeutic innovations have resulted in improved treatment strategies, with sustained abstinence rates of up to $50 \%$ reported for pharmacological interventions such as nicotine replacement in combination with behavior-change procedures. ${ }^{3}$ The twentieth

Based on an introduction to a panel discussion as part of a conference, "Tobacco Use: A Perspective for Alcohol and Drug Researchers", sponsored by the Addiction Research Foundation in Toronto, Canada on Thursday, 14 April 1988. report of the Surgeon General of the United States ${ }^{4}$ documents the progress that has been made.

The possibility of commonalities in patterns of substance use and abuse was noted some time ago. ${ }^{5}$ Among the similarities between responses to nicotine and alcohol, in particular, are the development of physiological dependence, with demonstrable tolerance following sustained use and withdrawal symptomology following discontinuation. Furthermore, behavioral dependence-stimulus control of smoking and drinking and persistent self-administration-has been shown for both substances. ${ }^{6}$ There is also evidence for the heritability of tobacco use $^{7}$ as well as of alcohol abuse. ${ }^{8}$ Consumption of both substances has been subject to regulation with respect to place and time, though both are legal in Western nations-alcohol for use in moderation and tobacco for those who "choose to participate in an adult custom'. Over the years, tobacco and beverage alcohol have spawned an extensive marketing and distribution network, and in the aggregate, each constitutes a multi-billion dollar industry.

An obvious and important difference between tobacco and alcohol use is in mode of administration. For nicotine, inhalation of cigarette smoke is the most common route, though other ways of dosing with nicotine have been employed over the years, involving intra-nasal administration (e.g. snuff) and buccal absorption (e.g. pipes, cigars, and smokeless tobacco). Alcohol use invariably involves consumption of a beverage. The difference between inhalation and drinking seems minor, but it may 
have profound consequences. Since nicotine has a relatively short half-life ( 90 to $120 \mathrm{~min}$ ) and since a bolus of nicotine can reach the brain in approximately $7 \mathrm{sec}$ via inhalation, smokers can acquire fairly precise dose regulation ('finger tip titration'). From the perspective of social learning theory, selfadministration of nicotine via inhalation involves a short delay of reinforcement and frequent dosing in a wide variety of stimulus contexts, providing the foundation for a behavioral pattern with great resistance to extinction.' Furthermore, inhalation allows the habitual smoker to minimize the aversive consequences of overdosing, a feature that is not trivial given nicotine's toxicity. Alcohol drinking, on the other hand, produces more lasting effects, and a dose sufficient to produce altered mood or performance persists several hours. Because alcohol taken orally reaches the brain more slowly, the drinker has less precise information about the amount ingested; consequently, there is greater likelihood of overdosing and aversive consequences.

Five to ten per cent of current smokers smoke occasionally (smoking 5 or fewer cigarettes/day); the remainder are regular smokers, most of whom are nicotine dependent. ${ }^{9}$ In contrast, the vast majority ( 80 to $90 \%$ or more) of alcohol consumers are occasional in their use and can be presumed nondependent. ${ }^{10}$ Nicotine has a dose-related biphasic effect, producing sympathomimetic, stimulant effects at low doses and muscle-relaxing and calming effects at higher doses. ${ }^{2}$ Although there are also dose-related biphasic effects with alcohol consumption, the overall impact-particularly at high doses-is sedation and impaired muscular co-ordination. An important, perhaps critical, difference between the two substances (relevant to the doseeffects just mentioned) is that nicotine administration can be adjusted to fit the circumstances. Thus, nicotine can be used to increase alertness and enhance performance or to reduce tension and relieve anxiety temporarily. For this perspective, nicotine administration via smoking may be the 'ideal' drug habit for a culture that values thrift and a strong work ethic: not only does smoking provide a brief respite from work with minimal interference with productivity, but, ironically, the habit even selects out older people through premature mortality, thereby anticipating their diminished productivity and increased utilization of expensive healthcare services.

While the introduction of tobacco to European culture was greeted with dismay in some quarters, ${ }^{11}$ a large number of people became devoted users over the years and a substantial industry developed (with revenues currently in excess of thirty billion dollars per annum in the United States). It is only 25 years ago that the devastating health consequences of smoking were widely publicized and even more recently that the extent of nicotine's dependenceproducing nature has been fully appreciated. ${ }^{4}$ Over a remarkably short space of time, smoking has gone from being seen as socially acceptable to being designated as highly undesirable and a public health menace. The tobacco industry has employed its considerable economic power in an attempt to divert this dramatic shift in public opinion, ${ }^{12}$ but so far, its efforts have been unsuccessful. The object of all this concern-the smoker-has been acquiescent and has generally complied with the new constraints and interdictions.

In contrast, perhaps because of its evident and immediate behavioral toxicity (disruption of ongoing behavior and pronounced shifts in mood) as well as the serious occupational and social impairment resulting from its chronic use, the drinking of alcohol has been a source of societal ambivalence and concern since antiquity, with some cultures seeking to limit excessive consumption and others -most notably Islam-attempting to prohibit it entirely. Throughout much of history, people with serious alcohol problems have been treated as social outcasts, and societies have at various times designated problem drinking as immoral, illegal, or irresponsible. In its modern form, societal ambivalence is reflected in disagreements between those who consider alcoholism a disease and those who define alcohol abuse as a disorder of self-control ('traditionalists' versus 'anti-traditionalists'), 13,14 and rancorous debates have erupted over various issues such as the object of treatment of alcoholism and problem drinking. ${ }^{15-17}$ Elements of society far removed from research, treatment, or management of substance abuse are being drawn into debate; recently, for example, in a case involving the Veterans Administration of the United States, the Supreme Court was asked to render an opinion on the disability status of former members of the armed forces with alcohol problems; the decision required a judgment about whether alcohol abuse is a conduct problem or a disease. ${ }^{18,19}$

Disagreements in the smoking and health field have been muted by comparison. For instance, in the late 1970's, arguments over the feasibility of developing 'safe' cigarettes arose, and the leadership of the Office on Smoking and Health (a U.S. Government agency involved in co-ordinating in- 
formation about the health hazards of smoking) was abruptly changed in response to public condemnation; the disputes died down after a few years, however, when the difficulties involved in accomplishing the task were more fully appreciated. Currently, the so-called 'smoking controversy' is largely a creation of media controlled by the tobacco industry, whose self-interest is clearly threatened by evidence suggesting that products that deliver nicotine are both addictive and harmful to health." As has been noted by Fingarette ${ }^{20}$ in his review of contrasting policies in the management of smoking and alcohol problems, considerable progress has been made in dealing with smoking. Policy recommendations and treatment methods generally operate in concert, and research is conducted primarily within a framework in which formal questions are posed, measurement techniques developed, observations made, scrutinized, and replicated, and the resulting evidence evaluated and disseminated.

The critical difference between the alcohol and nicotine fields seems to be in the existence of a vocal lay constituency made up of people with alcohol problems who, especially in the United States, have come to wield considerable political and economic power in the service of a particular perspective. Despite the fact that the disease concept of alcoholism was proposed by Jellinek ${ }^{21}$ simply as a 'working hypothesis' for guiding research, from the perspective of the people who speak for the fellowship of recovering alcoholics, the disease concept has begun to function as established fact. ${ }^{20}$ Research on the "genetics of alcoholism, neurochemistry of addition, and the search for biological interventions"13,14 is encouraged to the extent that it seems to support the idea that the alcoholic is unable to resist alcohol and that he/she must give up the illusion of control as the first step toward rehabilitation. It follows that investigations involving behavior modification and self-control, with their emphasis on behaviour and its consequences, are of questionable relevance and value. In practical terms, defense of the disease model is linked with protection against losing third party medical reimbursement, dismantling residential treatment facilities, and a possible return to the "moral concept of alcoholism" and "centuries of stigma". 13,14 While these concerns are understandable, an unfortunate by-product is the relegation of research to the task of affirming and bolstering the prestige of 'traditional wisdom' rather than generating new knowledge. The mission of science has been distorted, therefore, and its potential contribution, diminished.
In conclusion, the most obvious lesson that alcohol researchers can derive from smoking and other substance research is that, because of its history, various segments of the alcoholism field operate by very different rules. While nicotine selfadministration and smoking can serve as models for alcohol abuse in the laboratory, the commonality does not extend to alcohol problems in their current societal context. A large number of the presumed beneficiaries of alcoholism research (i.e. people with serious alcohol problems) have a fundamentally different perspective on the problem than do researchers. A possible explanation is that unlike smokers, alcoholics as a group were subject to ridicule, to criminal sanctions, and to moral censure. They were rejected by the sober majority and even by the helping professions. Now that alcohol abuse has become recognized as a larger social problem rather than just a character defect of isolated individuals, access to the medical system has been legitimized ${ }^{20}$ and new resources have been allocated for rehabilitation and research. As part of securing these gains, a call to arms has been issued against an apparent enemy, characterized as

"forces of wealth and cunning who promote behavioral retraining as a means to avoid the penalty of abstinence borne by those ... who have chosen the traditionalist route to recovery". ${ }^{23}$

Alcohol researchers will not be able to ignore the historical and epistomological differences between alcoholism and other substance abuse fields indefinitely. Progress will be difficult if not impossible until the 'traditionalist' and 'anti-traditionalist' perspectives on alcohol problems are reconciled and some common objectives are identified.

\section{References}

1. Pomerlanu, O. F. (1981) Underlying mechanisms in substance abuse: Examples from research on smoking, Addictive Behaviors, 6, pp. 187-196.

2. Pomerleau, O. F. \& Pomerleau, C. S. (1984) Neuroregulators and the reinforcement of smoking: towards a biobehavioral explanation, Neuroscience and Biobehavioral Reviews, 8, pp. 503-513.

3. Pomerleau, O. F. \& Pomerleau, C. S. (Eds) (1988) Nicotine Replacement: a critical evaluation (New York, Alan R. Liss).

4. USDHHS (1988) Health Consequences of Smoking: nicotine addition (Washington, D.C., United States Department of Health and Human Services).

5. KRASNEGOR, N. (Ed.) (1979) Behavioral analysis and treatment of substance abuse, NIDA Research Monograph 25 (Rockville, MD, National Institute on Drug Abuse). 
6. GoldBerg, S. R. EN STOLERMAN, I. P. (Eds) (1986) Behavioral Analysis of Drug Dependence (Orlando, FL, Academic Press).

7. Hughes, J.R. (1986) Genetics of smoking: a brief review, Behavior Therapy, 17, pp. 335-345.

8. ClONINGER, C. R. (1987) Neurogenic adaptive mechanisms in alcoholism, Science, 236, pp. 410-416.

9. Shiffman, S. Tobacco chippers: individual differences in tobacco dependence, Psychopharmacology, 97, pp. 539-547.

10. SCHUCKIT, M. A. (1985) Alcohol Patterns and Problems (New Brunswick, NJ, Rutgers University Press).

11. TAYLOR, P. (1985) The Smoke Ring: tobacco, money and multinational politics (New York, New American Library, Mentor Books).

12. Liebman, B. (1988) Tobacco titans get tough, Nutrition Action Newsletter, 15, pp. 8-9.

13. WALLACE, J. (1987) Waging the war for wellness, Part I, Professional Counselor, January/February, pp. 21-39.

14. WaLlaCe, J. (1987) Waging the war for wellness, Part II, Professional Counselor, March/April, pp. 21-27.
15. Pendery, M. L., Maltzman, I. M. \& West, L. J. (1982) Controlled drinking by alcoholics? New findings and a reevaluation of a major affirmative study, Science, 217, pp. 169-175.

16. SOBELL, M. B. \& SOBELL, L. C. (1973) Individualized behavior therapy for alcoholics, Behavior Therapy, 4, pp. 49-72.

17. Sobell, M. B. \& SobelL, L. C. (1984) The aftermath of heresy: A response to Pendery et al's (1982) critique of "individualized behavior therapy for alcoholics", Behaviour Research and Therapy, 22, pp. 413-440.

18. HoldeN, C. (1987) Is alcoholism a disease? Science, 238, p. 1647.

19. Supreme court denies plea of alcoholics vets, Science, 240, p. 597.

20. FINGARETTE, H. (1988) Heavy Drinking: The myth of alcoholism as a disease, pp. 134-135 (Berkeley, CA, University of California Press).

21. Jellinek, E. M. (1960) The Disease Concept of Alcoholism (New Haven, CT, Hillhouse Press).

22. Creager, C. (1987) Special people, special causes, Professional Counselor, January/February, p. 4. 
This document is a scanned copy of a printed document. No warranty is given about the accuracy of the copy. Users should refer to the original published version of the material. 